\title{
Prediction of pre-eclampsia: role of placental laterality by ultrasonography
}

\section{Shailesh Janardhan Kore*, Rohit Khot, Pradnya Supe, Divya Kanavia, Chaitra Thunga, Yogeshwar Nandanwar}

Department of Obstetrics and Gynaecology, L.T.M.M.C and L.T.M.G.H, Mumbai, Maharashtra, India

Received: 22 February 2016

Revised: 28 February 2016

Accepted: 18 March 2016

\section{*Correspondence:}

Dr. Shailesh Janardhan Kore,

E-mail: pradssupe@gmail.com

Copyright: (c) the author(s), publisher and licensee Medip Academy. This is an open-access article distributed under the terms of the Creative Commons Attribution Non-Commercial License, which permits unrestricted non-commercial use, distribution, and reproduction in any medium, provided the original work is properly cited.

\section{ABSTRACT}

Background: Pre-eclampsia, the most common medical complication of pregnancy, continues to be one of the leading causes of maternal morbidity and mortality. Women with mild and moderate degrees of gestational hypertension can often be treated conservatively and delivered at or near term with good perinatal outcome. The objective of this study was to find out whether placental laterality as determined by ultrasound done between $20-24$ weeks can be used as a predictor of development of preeclampsia.

Methods: In this prospective study, a total of 200 low risk singleton pregnant women attending the antenatal clinic at tertiary teaching institute were included. The location of the placenta was determined by ultrasound at 20-24 weeks. The women were divided into two groups- group A with Central placenta and group B with Lateral placenta. The end point of the study was the development of preeclampsia as per the ACOG criteria or delivery.

Results: Out of 200 antenatal women, 161 had central placenta (Group-A) and 39 had unilateral placenta (Group-B). A total of 32 women developed preeclampsia, of which $19(59.38 \%)$ had unilaterally located placenta at 20-24 weeks. This relationship was statistically highly significant $(\mathrm{P}<0.0001)$. The sensitivity, specificity, positive predictive value and negative predictive value of using placental laterality as a screening test were $59.38 \%, 88.10 \%, 48.72 \%$ and $91.93 \%$ respectively.

Conclusions: Placental laterality as determined by ultrasound at 20-24 weeks; is a simple yet reliable and cost effective predictive screening test for development of preeclampsia.

Keywords: Pre-eclampsia, Placental laterality, Ultrasonography

\section{INTRODUCTION}

Pre-eclampsia, the most common medical complication of pregnancy continues to be one of the leading causes of maternal morbidity and mortality. Women with mild and moderate degrees of gestational hypertension can often be treated conservatively and delivered at or near term with good perinatal outcome. However, severe cases often warrant iatrogenic premature delivery in view of maternal interests. This often means compromising on the baby, with increase in perinatal morbidity and mortality.
Various screening tests have been proposed for the prediction of preeclampsia with varying results. Some of these tests have shown potential as practical early screening test for the prediction of pre-eclampsia and related complications of pregnancy. Currently, there are no prospective studies or randomised trials which evaluate the benefits and risks of first trimester screening for prediction of pre eclampsia. ${ }^{1}$

In our study, we have made an attempt to analyse the efficacy of the placental location, rather laterality, as 
determined by ultrasonography done at 20-24 weeks in predicting women at risk of developing pre-eclampsia.

The objective of this study was to assess the value of lateral location of placenta as seen on ultrasonography at 20 weeks of gestation in predicting preeclampsia. And to analyse other parameters which can be combined with USG localization of placenta to improve sensitivity and specificity.

\section{METHODS}

A prospective study was conducted at single working unit of department of obstetrics and gynecology at major tertiary teaching institute in Mumbai, Maharashtra, India and included 200 low risk antenatal pregnant women attending outpatient department.

Tran's abdominal ultrasonography was done on these 200 women by curvilinear USG probe at 20 weeks of gestation. Based on the location of placenta they were classified into 2 groups. Women having central placenta (Equally/almost equally distributed between right and left side of the uterus, irrespective of anterior, posterior or fundal position of placenta) were categorized in group A, while women having Lateral Placenta (when $70 \%$ or more of placenta was on either side of the midline, Irrespective of anterior, posterior or fundal position of placenta) were in group B (Figure 1).
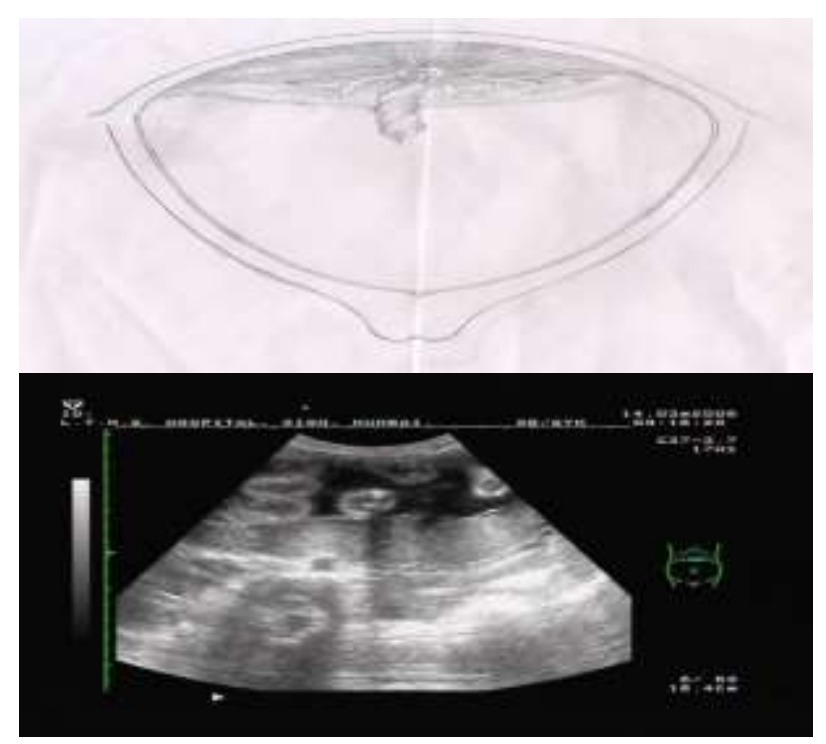

Figure 1: Central placenta-schematic diagram and USG image.

Results of the sonography were correlated with development or non-development of preeclampsia or gestational hypertension as per ACOG criteria. All women followed up in ANC and delivered at our hospital.

Women who did not develop hypertension were followed up to delivery.

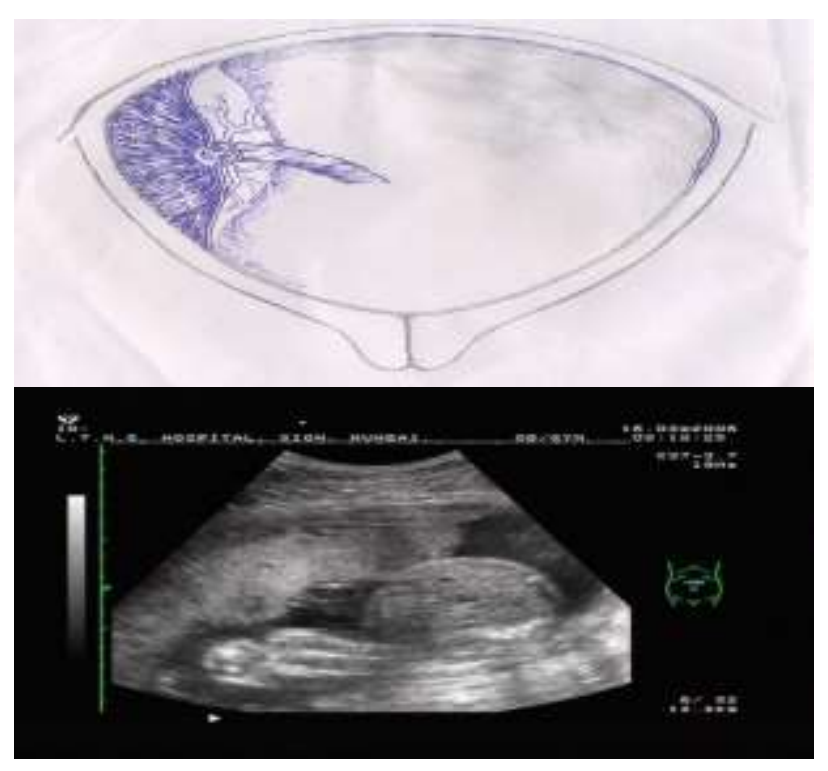

Figure 2: Lateral placenta-schematic diagram and USG image.

Women developing hypertension were managed according to severity and gestational age as per unit protocol.

Results in two groups were compared for statistical significance.

\section{RESULTS}

This Out of 200 woman, majority were below 30 years of age.

Table 1: Placental location and development of preeclampsia.

\begin{tabular}{|llll|}
\hline Location of Placenta & \multicolumn{2}{l}{ Development of } \\
& $\begin{array}{l}\text { Preeclampsia } \\
\text { Yes }\end{array}$ & No & \\
\hline Central (Group A) & 13 & 148 & 161 \\
\hline Lateral (Group B) & 19 & 20 & 39 \\
\hline Total & $\mathbf{3 2}$ & $\mathbf{1 6 8}$ & $\mathbf{2 0 0}$ \\
\hline
\end{tabular}

There were 87 primigravida and 113 multigravidas. According to location of placenta, women were divided into two groups, for analysis.

One sixty one women had a centrally situated Placenta and hence belonged to group A while 39 women had a laterally situated Placenta and belonged to group B.

All these women were regularly followed up in the Antenatal OPD.

Out of the 200 women, 32 developed preeclampsia/gestational hypertension giving an incidence of $16 \%$. Out of the 39 women with laterally located placenta, 19 developed preeclampsia while out of 
161 women with central placenta, only 13 women developed preeclampsia.

In other words $59.38 \%$ women who developed preeclampsia had a laterally situated placenta while $40.62 \%$ women had a centrally situated placenta $(\mathrm{P}<0.001)$.

Out of the 168 women who did not develop preeclampsia, 148 women $(88.10 \%)$ had a centrally situated placenta while just 20 women $(11.90 \%)$ had a laterally situated placenta $(\mathrm{p}<0.001)$.
When we combined all the three variables such as Age, Parity and Lateral location of placenta we can see that $100 \%$ of the primis with lateral placenta below 20 years developed preeclampsia (Table 2).

The sensitivity, specificity, positive predictive value and negative predictive value of this test were $59.38 \%$, $88.10 \%, 48.72 \%$ and $91.93 \%$ respectively.

The primigravida at extremes of ages having lateral placenta on ultrasound are clearly at high risk of developing preeclampsia.

Table 2: Age, parity and lateral location of placenta as predictors for preeclampsia in primigravida.

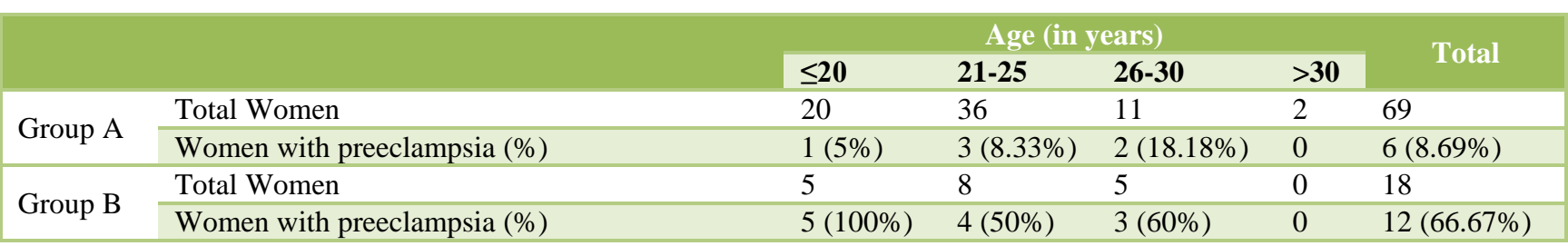

Table 3: Age, parity and lateral location of placenta as predictors for preeclampsia in multigravidas.

\begin{tabular}{|c|c|c|c|c|c|c|}
\hline & & \multicolumn{4}{|c|}{ Age (in years) } & \multirow{2}{*}{ Total } \\
\hline & & $\leq \mathbf{2 0}$ & 20-25 & 26-30 & $>\mathbf{3 0}$ & \\
\hline \multirow{2}{*}{ Group A } & Total Women & 5 & 50 & 27 & 10 & 92 \\
\hline & No of women with Preeclampsia (\%) & 0 & $4(8 \%)$ & $2(7.4 \%)$ & $1(10 \%)$ & $7(7.6 \%)$ \\
\hline \multirow{2}{*}{ Group B } & Total Women & 1 & 12 & 6 & 2 & 21 \\
\hline & No of women with preeclampsia (\%) & 0 & $4(33.33 \%)$ & $2(33.33 \%)$ & $1(50 \%)$ & $7(33.33 \%)$ \\
\hline
\end{tabular}

Table 4: Other screening tests for preeclampsia.

\begin{tabular}{|lllll|}
\hline Screening tests & Sensitivity (\%) & Specificity (\%) & PPV (\%) & NPV (\%) \\
\hline Mid-trimester BP estimation & 44 & 87 & 9 & 98 \\
\hline Urinary alb/creat. Ratio & 64 & 84 & 43 & 94 \\
\hline Hand grip test & 81 & 96 & 81 & 96 \\
\hline Platelet angiogenesis II binding & 50 & 88 & 60 & 84 \\
\hline Abnormal RI on Doppler & 47 & 81 & 44 & 83 \\
\hline Placental laterality (Our study) & 59.38 & 88.10 & 48.72 & 91.93 \\
\hline
\end{tabular}

It is thus clear that on combining parity with age and placental location the sensitivity of the test is increased.

In Multigravidas we can see that $50 \%$ with lateral placenta and age >30 years developed preeclampsia (Table 3).

Thus when all the three factors viz Age, Placental location and Parity are combined the sensitivity of the test is increased.

This is in concordance with the study conducted by Dekker and Sibai et al. ${ }^{2}$
IUGR was seen in 14 women with an incidence of $7 \%$. Out of these, seven women had laterally situated placenta giving an incidence of $17.95 \%$, while 7 had centrally situated placenta with an incidence of $4.35 \%(\mathrm{p}<0.001)$.

Thus $50 \%$ of women with IUGR had laterally situated placenta. The results are in concordance with study done by Lucy EG Kalanithi et al which concluded that IUGR pregnancies were nearly 4-fold more likely than nonIUGR pregnancies to have lateral placentation (either right or left) compared with anterior or posterior placentation. $^{3}$ 


\section{DISCUSSION}

Pre-eclampsia remains one of the leading causes of maternal and perinatal morbidity and mortality. Cases with mild and moderate degrees of pregnancy induced hypertension can be often continued till term with good maternal and perinatal outcome but severe cases warrant early intervention thus increasing perinatal morbidity and mortality.

We, therefore, need a test that would screen and detect women at high risk for developing PIH and pre-eclampsia so that prophylactic therapies can be started easily. Ideally, a screening test should be simple, inexpensive and reproducible, with high sensitivity and positive predictive value. To ensure maximum patient acceptability, it should be easy to perform and noninvasive. $^{4}$

It has been shown that in humans both uterine arteries have a significant number of branches and that each supply the corresponding side of the uterus. Although anastomoses between the two uterine arteries exist, there is no proof that these are functional. When the placenta is laterally located, the uterine artery closer to the placenta has lower resistance than the one opposite to it. In women with centrally located placentas both uterine arteries demonstrated similar resistance. ${ }^{5,6}$

When the placenta is centrally located, the uteroplacental blood flow needs are met by equal contribution from both uterine arteries. However when the placenta is laterally located, in the majority of cases, the uteroplacental blood flow needs are met primarily by one of the uterine arteries with minimal contribution from the other uterine artery via the collateral circulation. The degree of collateral circulation may not be the same in all women and deficient contribution may facilitate the development of preeclampsia, intrauterine growth retardation or both. The significance of normal placentation for this cytotrophoblastic invasion is high and the cytotrophoblasts fail to adopt a vascular adhesion phenotype in preeclampsia. This may explain the reduced trophoblastic invasion in laterally situated placenta when the uteroplacental blood flow needs are mainly met by one side uterine artery.

In our study, out of the 200 women, 32 developed Preeclampsia/Gestational hypertension giving an incidence of $16 \%$. Nineteen of these 32 cases had lateral placenta and 13 had central placenta.

Thus $59.38 \%$ women who developed preeclampsia had a laterally situated placenta while $40.62 \%$ women had a centrally situated placenta. Out of the 168 women who did not develop preeclampsia 148 women $(88.10 \%)$ had a centrally situated placenta while just 20 women $(11.90 \%)$ had a laterally situated placenta. The sensitivity, specificity, positive predictive value and negative predictive value were $59.38 \%, 88.10 \%, 48.72 \%$ and
91.93\% respectively. The sensitivity and specificity was much higher in primigravida and younger age group women. In the study conducted by Pai the results were $73 \%, 86 \%, 51 \%$ and $94 \%$ respectively which were comparable to our study. ${ }^{6}$

The results are also in accordance with the study of Kofinas et al in whom the sensitivity of the test was $73 \%{ }^{6}$ In our study placental laterality has a sensitivity of $59.38 \%$ which though low is much better than most other tests (Table 4). Also the Negative predictive Value and the Specificity are much higher as compared to the other tests. Also, if we combine this test with risk factors like prim parity and extremes of maternal age, the sensitivity is very high. Besides, it has a very good specificity and negative predictive value.

A number of other screening tests are being advocated for predicting the development of preeclampsia. We compared the performance of placental laterality as a screening test with some of the other tests as mentioned by Chan et al (Table 4). ${ }^{7}$

The results are comparable. As yet there is no practical, acceptable and reliable screening test for preeclampsia that has been thoroughly tried and tested. Though in last two decades, uterine artery Doppler velocimetry has been advocated as effective screening method for prediction of preeclampsia it requires expertise, is not easily available and is costly. Majority of pregnant women undergo an ultrasonography at around 18-20 weeks to rule out congenital anomalies. Placental localization in the same settings does not add to the cost of screening procedure. Thus it is noninvasive, easy to perform, easily available, reliable and cheap.

\section{CONCLUSIONS}

Placental laterality as determined by ultrasound at 20 weeks; alone or with age and parity is a simple yet reliable and cost effective predictive screening test for development of preeclampsia, and should be offered to all pregnant women attending antenatal clinic.

Funding: No funding sources Conflict of interest: None declared

Ethical approval: The study was approved by the Institutional Ethics Committee

\section{REFERENCES}

1. Sibai BM. First-trimester screening with combined maternal clinical factors, biophysical and biomarkers to predict preterm pre-eclampsia and hypertensive disorders: are they ready for clinical use? BJOG. 2015;122(3):282-3.

2. Dekker G, Sibai B. Primary, secondary, and tertiary prevention of pre-eclampsia. Lancet. 2001;357:209-15. 
3. Kalanithi LE, Illuzzi JL, Nossov VB, Frisbaek Y, Razeq SA, Copel JA, et al. Intrauterine growth restriction and placental location. J Ultrasound Med. 2007;26(11):1481-9.

4. Studd J, Baker F. Screening tests for pregnancy induced hypertension in Progress in obstetrics and Gynecology.1990;10:69.

5. Campbell S, Pearce JM, Hackett G, Overbeek TC, Hernandez C. Qualitative assessment of uteroplacental blood flow: early screening test for high risk pregnancies. Obstet Gynecol. 1986;68(5):649-53.

6. Kofinas AD, Penry M, Swain M, Hatjis CG. Effect of placental laterality on uterine artery resistance and development of preeclampsia and intra uterine growth retardation. Am J Obstet Gynecol. 1989;161:153-69.

7. Chan FY, Pun TC, Lam C, Khoo J, Lee CP, Lam YH. Pregnancy screening by uterine artery Doppler velocimetry- which criterion performs best ? Obstet Gynecol. 1995;85:596-602.

Cite this article as: Kore $\mathrm{SJ}$, Khot $\mathrm{R}$, Supe $\mathrm{P}$, Kanavia D, Thunga C, Nandanwar Y. Prediction of pre-eclampsia: role of placental laterality by ultrasonography. Int J Reprod Contracept Obstet Gynecol 2016;5:1433-7. 